\title{
Distribuição de Glyphosate e ACÚMUlo de Nutrientes em Biótipos DE AZEvÉM ${ }^{1}$
}

\author{
Glyphosate Distribution and Nutrient Accumulation in Ryegrass Biotypes
}

\author{
FERREIRA, E.A. ${ }^{2}$, SILVA, A.A. ${ }^{3}$, REIS, M.R. ${ }^{4}$, SANTOS, J.B. ${ }^{5}$, OLIVEIRA, J.A. ${ }^{6}$, VARGAS, L. ${ }^{7}$, \\ KHOURI, K.R. ${ }^{8}$ e GUIMARÃES, A.A. ${ }^{9}$
}

\begin{abstract}
RESUMO - Objetivou-se neste trabalho avaliar o acúmulo de nutrientes e a translocação de glyphosate em biótipos de azevém. Para isso, foram montados dois ensaios: no primeiro aplicou-se ${ }^{14} \mathrm{C}$-glyphosate, adicionando $10 \mu \mathrm{L}$ da calda sobre a face adaxial da primeira folha com ligula totalmente visivel, quando as plantas de azevém apresentavam três perfilhos. A quantidade de glyphosate absorvido, translocado e exsudado foi avaliada 64 horas após aplicação, por meio da medição da radiação emitida pelo ${ }^{14} \mathrm{C}$-glyphosate, em espectrômetro de cintilação liquida. O glyphosate foi quantificado em folha de aplicação, perfilhos, raizes e na solução nutritiva onde foram cultivados os biótipos de azevém. No segundo experimento, aplicou-se o glyphosate (480 $\left.\mathrm{g} \mathrm{ha}^{-1}\right)$ tanto no biótipo sensivel quanto no resistente. Após dez dias da aplicação, a parte área e as raizes das plantas foram coletadas e secas em estufa, sendo determinados os teores de macronutrientes. No primeiro ensaio, verificouse exsudação radicular em ambos os biótipos, nos quais a quantidade de glyphosate exsudada foi semelhante, não ultrapassando $5 \%$ do total que penetrou na planta. No perfilho principal do biótipo sensivel, comparado ao resistente, foi observada maior concentração do produto marcado. O biótipo resistente apresentou maior acúmulo de produto marcado na folha de aplicação; no sensivel, a maior parte do glyphosate foi encontrada nas raizes. Com relação ao segundo ensaio, na presença de herbicida o biótipo sensivel apresentou menor teor de fósforo tanto na parte aérea quanto na planta. Os biótipos resistente e sensivel, sem aplicação de herbicida, tenderam a apresentar maiores teores de $\mathrm{N}$ total e $\mathrm{N}$ inorgânico na parte aérea e na planta como um todo, quando comparados aos tratamentos em que foi realizada a aplicação do produto. Ambos os biótipos mostraram a mesma capacidade de absorção e acúmulo de macronutrientes na ausência do produto.
\end{abstract}

Palavras-chave: ${ }^{14} \mathrm{C}$-glyphosate, Lolium multiflorum, nutrição mineral, resistência.

\begin{abstract}
This work aimed to evaluate nutrient accumulation and glyphosate translocation in ryegrass biotypes. Two trials were conducted: in the first, $10 \mathrm{uL}$ of ${ }^{14} \mathrm{C}$-glyphosate ware applied on the upper side of the first leaf showing completely visible ligule, with ryegrass plants at the three-tiller stage. The amount of glyphosate absorbed, translocated and exudated was evaluated 64 hours after application, by using a liquid scintillation spectrometer. Glyphosate was quantified in the leaf it had been applied, in the tillers, roots and in the nutritive solution the plants were grown. In the second trial, glyphosate was applied on the resistant and the susceptible biotypes at the rate of $480 \mathrm{~g} \mathrm{~L}^{-1}$. Ten days after application, the shoot and roots were collected and ovendried and the levels of macronutrients were determined. In the first trial, root exudation was observed in both biotypes, in which the amount of exudated glyphosate was similar and not
\end{abstract}

1 Recebido para publicação em 28.4.2007 e na forma revisada em 4.1.2008

2 Pós-doutorando do Dep. de Fitotecnia da Universidade Federal de Viçosa - DFT/UFV, 36570-000, Viçosa-MG, <evanderalves@yahoo.com.br>; ${ }^{3}$ D.S., Professor do Dep. de Fitotecnia da Universidade Federal de Viçosa - DFT/UFV; ${ }^{4}$ EngoAgr ${ }^{\circ}$, Doutorando em Fitotecnia da Universidade Federal de Viçosa - DFT/UFV; ${ }^{5}$ D.S., Professor de Agronomia da FAAG/ Univale. Rua Israel Pinheiro, 2000, Bairro Universitário, 35020-220, Governador Valadares-MG; ${ }^{6}$ D.S., Professor do Departamento de Biologia Geral DBG-UFV; ${ }^{7}$ Pesquisador da Embrapa Trigo, Caixa Postal 451,99001-970, Passo Fundo-RS; ${ }^{8}$ Engo-Agr ${ }^{0}$, Mestre em Fitotecnia; ${ }^{9}$ Acadêmica de Agronomia/UFV.

Planta Daninha, Viçosa-MG, v. 26, n. 1, p. 165-173, 2008 
greater than 5\% of the absorbed glyphosate. In the main tiller of the susceptible biotype, a higher concentration of ${ }^{14} \mathrm{C}$-glyphosate was observed, compared to the resistant one. The resistant biotype showed more glyphosate accumulation in the leaf it had been applied and in the susceptible biotype; most ${ }^{14}$-C glyphosate was found in the root system. In the second trial, the susceptible biotype showed less $P$ accumulation than the resistant one. Both the resistant and susceptible biotypes tended to accumulate more total $N$ and inorganic $N$ in the shoot and in the whole plant in the absence of glyphosate, compared to the applied treatments. Both biotypes presented the same absorption capacity and macronutrients accumulation in the absence of the glyphosate.

Keywords: ${ }^{14} \mathrm{C}$-glyphosate, Lolium multiflorum, mineral nutrition, resistance.

\section{INTRODUÇÃO}

Há décadas os herbicidas tornaram-se insumos indispensáveis na agricultura, em razão de seu custo, sua eficiência e sua rapidez de operação para controle de plantas daninhas (Silva et al., 2007). No entanto, devido ao uso inadequado desses produtos, foram selecionados diversos biótipos de plantas daninhas resistentes a vários deles (Burnside, 1992).

Uma das grandes preocupações, depois de constatada a resistência, é desenvolver técnicas de manejo dessas plantas nas lavouras e, principalmente, evitar a dispersão delas para novas áreas (Christoffoleti \& Lopez-Ovejero, 2003). Todavia, para desenvolver novas técnicas de manejo é necessário conhecer as causas que levaram os biótipos a adquirir resistência, bem como conhecer a capacidade competitiva desses novos biótipos em relação àqueles que não apresentam resistência.

Dentre as possiveis causas de resistência, destacam-se as alterações na enzima EPSPs, o metabolismo diferencial do glyphosate ou o aparecimento de barreiras que dificultam a absorção, translocação e exsudação desses herbicidas (Christoffoleti \& Lopez-Ovejero, 2003).

Quanto à capacidade competitiva dos biótipos resistentes em relação aos sensiveis, a capacidade de extração e acúmulo de forma eficiente dos nutrientes pelas plantas pode ser fator importante na capacidade competitiva dos diferentes biótipos.

O glyphosate tem sido usado intensivamente na agricultura há mais de 25 anos e, até o momento, em poucas populações de plantas daninhas ocorreu pressão de seleção suficiente para aumento de biótipos resistentes. O primeiro caso de resistência de plantas daninhas a esse herbicida foi registrado em 1996. Nos últimos anos, devido ao uso repetido do glyphosate na mesma safra, têm-se observado ocorrências de espécies com biótipos resistentes em oito países, sendo elas: Lolium rigidum, Amaranthus palmeri, Amaranthus rudis, Ambrosia artemisiifolia, Eleusine indica, Conyza canadensis, Lolium multiflorum, Conyza bonariensis, Euphorbia heterophylla, Sorghum alepense e Plantago lanceolata (Weed Science, 2007).

O primeiro caso de Lolium multiflorum resistente ao glyphosate foi relatado por Perez \& Kogan (2002). O biótipo resistente foi identificado em pomares no Chile que vinham recebendo, em média, três aplicações de glyphosate por ciclo durante vários anos. No Brasil foram registrados até o momento quatro casos de plantas daninhas resistentes ao glyphosate: Lolium multiflorum (azevém), Coniza bonariensis (buva), Coniza canadensis (buva) e Euphorbia heterophylla (leiteiro). Biótipos de azevém resistentes ao glyphosate constituem um grave problema nas lavouras de soja transgênica e em pomares no Rio Grande do Sul, levando a considerável aumento nos custos de produção (Vargas, 2005).

Além do problema do aparecimento de resistência de plantas daninhas, outras modificações podem ocorrer em seu metabolismo, aumentando ou diminuindo suas atividades fisiológicas, que podem afetar seu crescimento e desenvolvimento. Desse modo, podem ocorrer 
alterações no status nutricional das plantas cultivadas. Trabalhos desenvolvidos com a cultura do arroz e mostarda por Mishra \& Kurchana (2001) e Rana (2000) evidenciaram alterações dos teores de macronutrientes em tecidos foliares devido à aplicação de herbicidas. Também Carolus et al. (1971) aplicaram diversos herbicidas em ervilhas e encontraram alteração nos teores de nutrientes com variação mínima de peso de matéria seca, enquanto Eastin \& Davies (1967) aplicaram atrazine em amendoim e constataram aumento nos teores de algumas formas de N. A aplicação de glyphosate para controle de algumas espécies de gramíneas, em áreas de cultivo de pínus sob estudo de competição entre a cultura e as plantas daninhas, aumentou em $31 \%$ a concentração foliar de K (Sword et al., 1998).

Neste trabalho foram avaliadas a absorção, translocação e exsudação do glyphosate por biótipos resistente e sensivel ao herbicida, assim como o efeito deste sobre a capacidade de acúmulo de nutrientes nesses biótipos.

\section{MATERIAL E MÉTODOS}

O presente trabalho constou de dois ensaios. No primeiro, plantas de azevém (biótipos resistente e sensivel) foram cultivadas em solução nutritiva, em câmara de crescimento com temperatura dia/noite de $25 / 16^{\circ} \mathrm{C}$, respectivamente, e fotoperiodo de 12 horas de luz. $\mathrm{O}{ }^{14} \mathrm{C}$-glyphosate foi aplicado em mistura com glyphosate comercial (480 $\left.\mathrm{g} \mathrm{ha}^{-1}\right)$ sobre a face adaxial da primeira folha com ligula totalmente visivel, utilizando-se uma microsseringa, adicionando-se $10 \mu \mathrm{L}$ da calda com atividade especifica de aproximadamente $700 \mathrm{~Bq}$, quando as plantas apresentavam três perfilhos aos 30 dias após a emergência.

A avaliação da absorção e translocação do ${ }^{14} \mathrm{C}$-glyphosate em biótipos resistente e sensível foi realizada 64 horas após o tratamento com o produto. Decorrido esse período, foram coletadas e fracionadas em folha de aplicação, perfilho principal, perfilho 2 e perfilho 3 e raízes, sendo avaliada também a solução nutritiva onde foram cultivadas as plantas. A folha de aplicação foi lavada com 9,0 mL de metanol, para extração do produto não-absorvido. Todo o material vegetal foi seco em estufa com circulação forçada de ar a $50^{\circ} \mathrm{C}$, por 48 horas, para obtenção da massa seca de cada parte da planta. Em seguida, procedeu-se à moagem de cada parte da planta em moinho de bola, até uma textura equivalente a 200 mesh. Depois disso, amostras de $100 \mathrm{mg}$ de massa seca de cada parte da planta (folha de aplicação, parte aérea e raízes) foram adicionadas em frascos de $20 \mathrm{~mL}$, para contagem. Dentro de cada frasco, as amostras foram homogeneizadas com $1 \mathrm{~mL}$ de Triton-X-100 por 14 segundos, adicionando-se, em seguida, $5 \mathrm{~mL}$ de coquetel de cintilação, sendo novamente submetidas à homogeneização. Os frascos assim preparados foram colocados em espectrômetro de cintilação líquida Beckman 6500, o qual corrigia o quenching automaticamente.

Os valores da radiação (cpm total) encontrada na solução de lavagem e na planta foram convertidos em porcentagem em relação à radiação total medida. Na planta, esses valores de ${ }^{14} \mathrm{C}$-glyphosate foram convertidos em porcentagem em relação ao total presente na planta, distribuídos em folha de aplicação, perfilhos, raízes e exsudação radicular. Os resultados foram apresentados em gráficos, utilizando-se médias observadas e desvios-padrões.

O segundo ensaio foi instalado e conduzido em casa de vegetação, utilizando vasos com capacidade de $6 \mathrm{~L}$ de substrato, composto de Argissolo Vermelho-Amarelo (Tabela 1) e areia lavada na proporção de 3:1, e adubado. Os vasos foram mantidos em ambiente protegido e irrigados diariamente, visando a manutenção da umidade adequada do solo, sendo mantidas três plantas por vaso. $O$ experimento foi disposto em um esquema fatorial $2 \times 2$, sendo os tratamentos compostos pelas parcelas que receberam aplicação de glyphosate $(\mathrm{C} / \mathrm{H})$ e não receberam aplicação de glyphosate $(\mathrm{S} / \mathrm{H})$ e pelos biótipos resistente e sensivel arranjados em blocos ao acaso (DBC), com quatro repetições.

Aos 45 dias após a emergência (DAE) foi aplicado o herbicida glyphosate na dose de $480 \mathrm{~g} \mathrm{ha}^{-1}$ da formulação comercial Roundup Transorb $^{\circledR}$, contendo o sal de isopropalamina.

Dez dias após aplicação dos tratamentos, as plantas foram colhidas, fracionadas em parte área e raízes e secas em estufa com circulação forçada de ar à temperatura de $65{ }^{\circ} \mathrm{C}$. As amostras foram transferidas para o Laboratório 
Tabela 1 - Composição química e textural do solo utilizado no experimento. Viçosa-MG, 2006

\begin{tabular}{|c|c|c|c|c|c|c|c|c|c|c|}
\hline \multicolumn{11}{|c|}{ Análise química } \\
\hline $\mathrm{pH}$ & $\mathrm{P}$ & $\mathrm{K}^{+}$ & $\mathrm{H}+\mathrm{Al}$ & $\mathrm{Al}^{3+}$ & $\mathrm{Ca}^{2+}$ & $\mathrm{Mg}^{2+}$ & $\mathrm{CTC}_{\text {total }}$ & $\mathrm{V}$ & $\mathrm{M}$ & $\mathrm{MO}$ \\
\hline $\mathrm{H}_{2} \mathrm{O}$ & \multicolumn{2}{|c|}{$---\mathrm{mg} \mathrm{dm}^{-3}---$} & \multicolumn{5}{|c|}{$\begin{array}{ll}-- \\
-\end{array}$} & \multicolumn{2}{|c|}{----- \% ----- } & -- dag kg $\mathrm{kg}^{-1}-$ \\
\hline 5,10 & 235,8 & 115 & 5,50 & 0,10 & 8,39 & 0,74 & 14,92 & 63,10 & 1,10 & 4,18 \\
\hline \multicolumn{11}{|c|}{ Fração mineral (\%) } \\
\hline \multicolumn{2}{|c|}{ Areia fina } & \multicolumn{2}{|c|}{ Areia grossa } & & \multicolumn{2}{|c|}{ Silte } & \multicolumn{2}{|l|}{ Argila } & \multicolumn{2}{|c|}{ Textura } \\
\hline \multicolumn{2}{|c|}{10} & \multicolumn{2}{|r|}{36} & & \multicolumn{2}{|l|}{5} & \multicolumn{2}{|l|}{49} & \multicolumn{2}{|c|}{ Argilo-Arenosa } \\
\hline
\end{tabular}

de Nutrição Mineral, onde foram moídas e submetidas à digestão nítrico-perclórica. Utilizaram-se amostras de 0,5 g em $10 \mathrm{~mL}$ de $\mathrm{HNO}_{3}$ concentrado, submetidas à temperatura de no máximo $200^{\circ} \mathrm{C}$, para a digestão. Obtido o extrato, foram determinados os teores de fósforo $(\mathrm{P})$, enxofre $(\mathrm{S})$, potássio $(\mathrm{K})$, cálcio $(\mathrm{Ca})$ e magnésio $(\mathrm{Mg})$. Para determinação do nitrogênio orgânico (N orgânico), as amostras foram submetidas à digestão sulfúrica. Foi determinada também a concentração total de nutrientes na planta (teor de nutriente $x$ massa seca da planta).

Os dados foram submetidos à análise de variância, e a comparação entre as médias foi feita pelo teste $\mathrm{F}$ a $5 \%$ de significância.

\section{RESULTADOS E DISCUSSÃO}

No primeiro ensaio, foi observada maior concentração de ${ }^{14} \mathrm{C}$-glyphosate na folha de aplicação do biótipo resistente (68\%). No biótipo sensivel, a folha de aplicação apresentou $40 \%$ do produto marcado (Figura 1). Resultados semelhantes foram encontrados por Ferreira et al. (2006), os quais verificaram, depois de 64

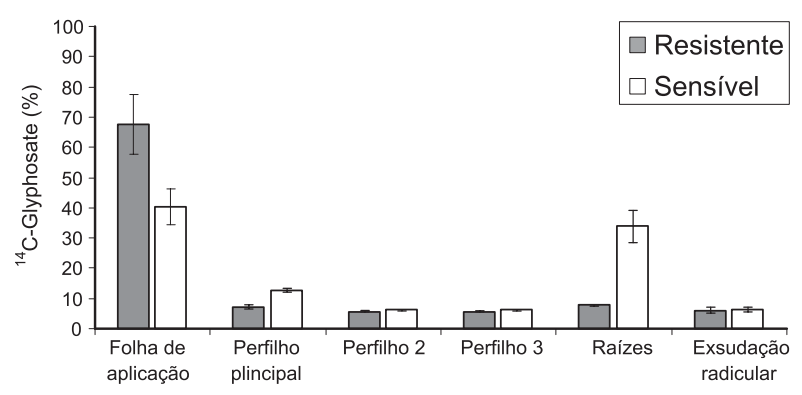

Figura 1 - Porcentagem de ${ }^{14} \mathrm{C}$-glyphosate presente na folha principal, perfilhos 2 e 3 , raízes e exsudação radicular em biótipos de azevém resistente e sensível ao glyphosate, 64 horas após aplicação do produto. horas da aplicação do produto, que aproximadamente $81 \%$ do total absorvido se encontrava retido na folha onde foi realizada a aplicação, enquanto no biótipo sensível a retenção foi de $54 \%$.

No perfilho principal, onde foi realizada a aplicação do produto, observou-se maior acúmulo de ${ }^{14} \mathrm{C}$-glyphosate no biótipo sensivel: aproximadamente $15 \%$ do total encontrado na planta; esse valor, no biótipo resistente, foi de aproximadamente $10 \%$. Para os perfilhos 2 e 3 esses valores foram semelhantes (Figura 1). A maior concentração do produto marcado foi encontrada na parte aérea do biótipo sensível $25 \%$, enquanto no resistente esse valor foi de cerca de $20 \%$ (Figura 2 ).

Nas raizes, a distribuição do ${ }^{14} \mathrm{C}$-glyphosate foi consideravelmente diferente entre os dois biótipos: no sensivel, a concentração do produto marcado foi cerca de quatro vezes maior que no resistente (40 e 10\%, respectivamente). Não foi observada diferença com relação à exsudação radicular nos dois biótipos; os valores não ultrapassaram 5\% (Figura 1). Rodrigues et al. (1982) observaram exsudação radicular

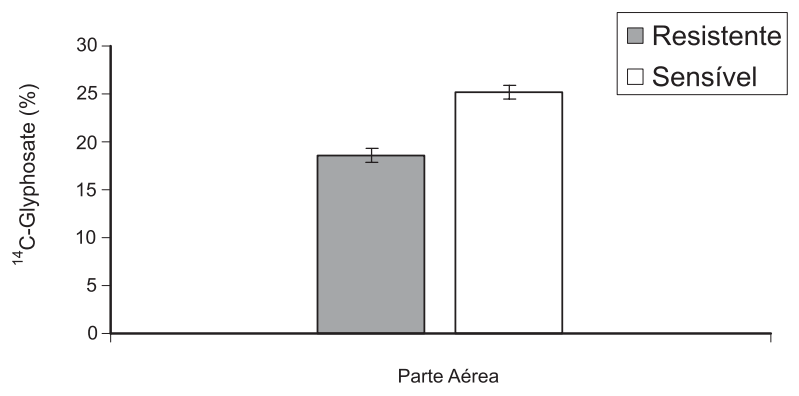

Figura 2 - Porcentagem de ${ }^{14} \mathrm{C}$-glyphosate presente na parte aérea em biótipos de azevém resistente e sensível ao glyphosate, 64 horas após aplicação do produto. 
de ${ }^{14} \mathrm{C}$-glyphosate por plantas de trigo tratado com este herbicida e que plantas de milho cultivadas próximo ao trigo absorviam o ${ }^{14} \mathrm{C}$-glyphosate pelas raízes, distribuindo-o por todas as partes da planta, sendo as maiores concentrações encontradas nas raízes. É provável que, nesse período de dez dias, parte do glyphosate já tenha sido metabolizada a ácido aminometilfosfônico (AMPA). A principal rota de degradação do glyphosate em plantas de soja, denominada rota do AMPA, leva à formação do próprio aminometil ácido fosfônico e glioxilato, a partir do glyphosate. O AMPA, uma vez formado, é clivado em metilamina e fosfato inorgânico, como produtos finais de degradação; todas essas reações podem ocorrer na planta, porém é o solo o principal local da degradação do glyphosate realizada por microrganismos (Hoagland, 1980).

No segundo ensaio, os biótipos resistente e sensivel de azevém apresentaram maiores teores de $\mathrm{N}$ total na parte aérea quando submetidos ao tratamento com glyphosate $(33,82$ e $35,42 \mathrm{~g} \mathrm{~kg}^{-1}$ nas plantas tratadas, contra 28,64 e $27,00 \mathrm{~g} \mathrm{~kg}^{-1}$ nas plantas não tratadas com o produto, respectivamente para os biótipos resistente e sensivel), conforme Tabela 2. Sword et al. (1998) constataram redução nos teores de $\mathrm{N}$ total nas folhas de plantas de pínus que receberam o glyphosate em condições de competição. No tocante ao teor de N orgânico, não foi observada diferença na concentração do produto tanto entre os biótipos quanto na presença de herbicida. Santos et al. (2007), trabalhando com soja RR, observaram redução nos teores foliares de $\mathrm{N}$ total após aplicação de diferentes formulações de glyphosate.

No biótipo sensivel, as plantas que receberam tratamento herbicida apresentaram três vezes mais $\mathrm{N}$ inorgânico, comparado ao biótipo resistente. Neste biótipo, o tratamento com herbicida apresentou duas vezes mais $\mathrm{N}$ inorgânico que o tratamento que não recebeu o produto (16,83 e $8,58 \mathrm{~g} \mathrm{~kg}^{-1}$, respectivamente), porém não foi observada diferença nos teores de $\mathrm{N}$ inorgânico entre os biótipos (Tabela 2). Deve-se considerar que o nitrogênio é um elemento constituinte de moléculas de proteínas, coenzimas, ácidos nucléicos e citocromos, provocando grande resposta nas plantas quando aplicado, e o glyphosate é derivado de aminoácidos, sendo seu mecanismo de ação a inibição da enol-piruvil-shikimato-fosfato sintetase (EPSPs), enzima responsável por uma das etapas da sintese dos aminoácidos aromáticos fenilalamina, tirosina e triptofano (Hess, 1994). Sem a síntese desses aminoácidos a formação de proteínas é comprometida, além da paralisa-

Tabela 2 - Teor de macronutrientes na parte aérea dos biótipos de azevém (L. multiflorum) resistentes e sensíveis ao glyphosate, aos $10 \mathrm{DAT}(\mathrm{C} / \mathrm{H}=$ com herbicida; $\mathrm{S} / \mathrm{H}=$ sem herbicida $)$

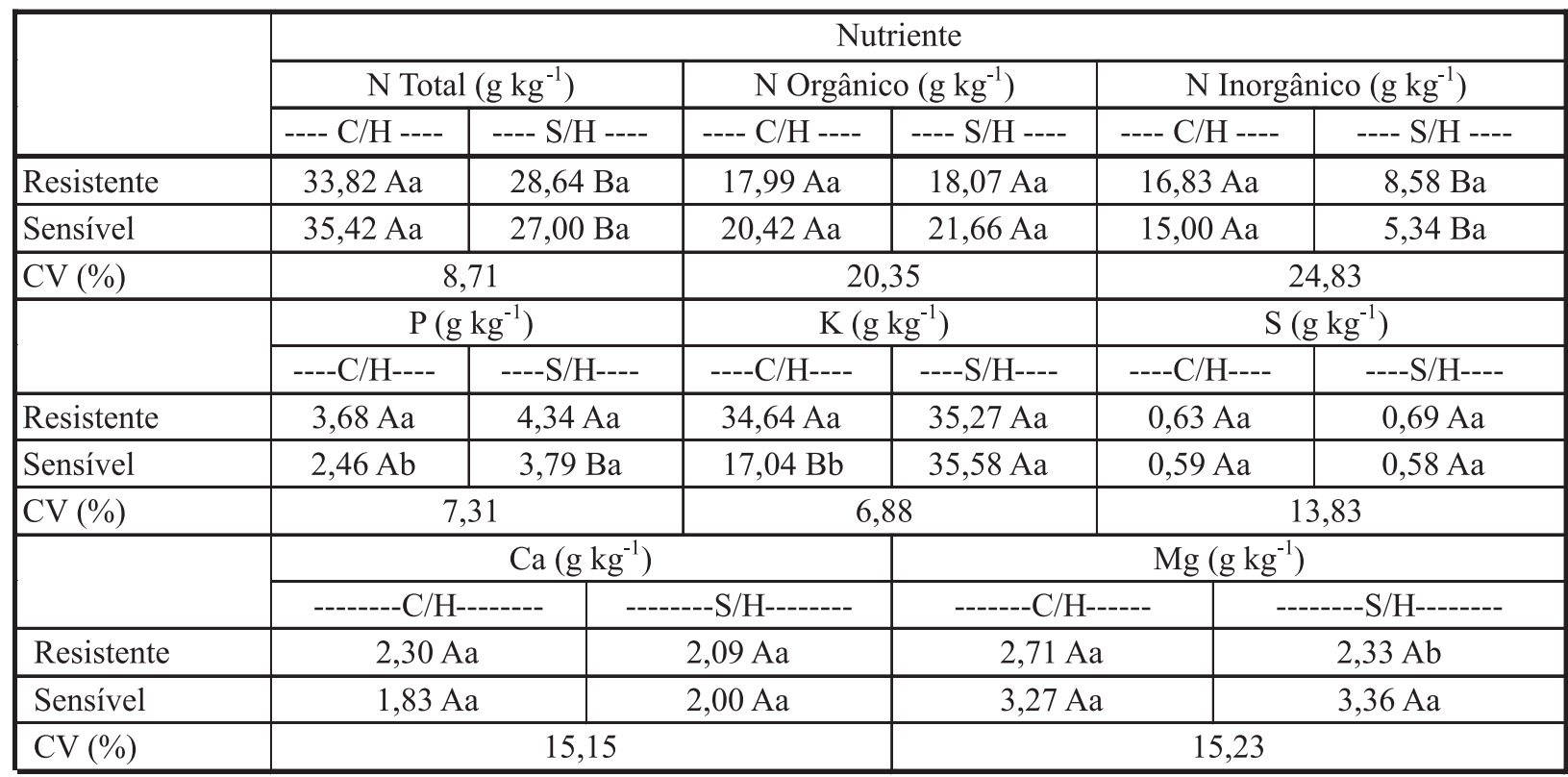

Médias seguidas por mesma letra, maiúscula na linha e minúscula na coluna, não diferem entre si pelo teste $\mathrm{F}$ a $5 \%$ de probabilidade. 
ção da sintese de compostos secundários, como vitaminas, hormônios e substâncias fenólicas (Kissmann, 2003). Dessa forma, o maior teor de $\mathrm{N}$ inorgânico no biótipo sensivel pode ser devido à inibição da enzima EPSPs e à paralisação da síntese de aminoácidos. O acúmulo de $\mathrm{N}$ inorgânico indica que a enzima nitrato redutase pode estar sendo afetada pelo herbicida.

O biótipo sensível tratado com glyphosate apresentou menor teor de fósforo na parte aérea $\left(2,46 \mathrm{~g} \mathrm{~kg}^{-1}\right)$ quando comparado ao tratamento sem herbicida $\left(3,79 \mathrm{~g} \mathrm{~kg}^{-1}\right)$. Quando se comparam os dois biótipos, verifica-se maior teor de $\mathrm{P}$ para o biótipo resistente, independentemente da aplicação do herbicida em ambos os tratamentos (Tabela 2). O P assume importantes funções nos tecidos vegetais, como componente de fosfatos, açúcares, ácidos nucléicos, nucleotídeos, coenzimas, fosfolipídios e ácido fitico, e tem papel central em reações que envolvem ATP (Taiz \& Zeiger, 2002). Com relação ao $\mathrm{K}$, foi constatado também menor teor desse nutriente na parte aérea do biótipo sensivel que recebeu a aplicação do glyphosate $\left(17,04 \mathrm{~g} \mathrm{~kg}^{-1}\right)$, quando comparado ao tratamento que não recebeu o herbicida $\left(35,58 \mathrm{~g} \mathrm{~kg}^{-1}\right)$, conforme Tabela 2 . O K é requerido como co-fator de mais de 40 enzimas e é o principal cátion no estabelecimento do turgor celular e na manutenção da eletroneutralidade celular (Taiz \& Zeiger, 2002). É possivel que tanto para o $\mathrm{P}$ quanto para o K ocorra redução na absorção desses nutrientes pelo biótipo sensivel tratado com o produto. Não foram observadas diferenças entre os biótipos nos teores de $\mathrm{S}$, Ca e Mg (Tabela 2).

Ao avaliar os teores de macronutrientes nas raízes, não foram constatadas diferenças entre os biótipos resistente e sensivel, assim como entre os tratamentos com e sem herbicida (Tabela 3).

A avaliação do teor total de nutrientes na planta indicou menor acúmulo de $\mathrm{N}$ total no biótipo sensivel não tratado com glyphosate, comparado aos biótipos resistente e sensivel tratados (Tabela 4). Já para P e K observou-se comportamento diferente: o biótipo sensivel tratado apresentou menor teor total desses nutrientes. O teor de P no biótipo sensivel tratado foi de $2,97 \mathrm{~g} \mathrm{~kg}^{-1}$, e nas plantas que não receberam o tratamento, de $4,41 \mathrm{~g} \mathrm{~kg}^{-1}$; no biótipo resistente não foi observada diferença entre as plantas tratadas e não tratadas com o produto, indicando que o glyphosate não interfere na absorção de P no biótipo resistente. Com relação ao $\mathrm{K}$, no biótipo sensivel tratado foi constatado menor teor do nutriente: $40,43 \mathrm{~g} \mathrm{~kg}^{-1}$ contra $70,14 \mathrm{~g} \mathrm{~kg}^{-1}$ nas plantas não tratadas. Os resultados referentes aos teores de $\mathrm{N}$ total, $\mathrm{P} \mathrm{e}$ $\mathrm{K}$ na planta são similares aos observados na parte aérea dos biótipos. Para os macronutrientes $\mathrm{S}$, Ca e Mg não foram observadas diferenças entre os biótipos resistente e sensivel nos tratamentos com e sem herbicida (Tabela 4).

O biótipo sensível, na ausência de herbicida, apresentou maior acúmulo de massa seca

Tabela 3 - Teor de macronutrientes nas raízes dos biótipos de azevém (L. multiflorum) resistentes e sensíveis ao glyphosate, aos $10 \mathrm{DAT}(\mathrm{C} / \mathrm{H}=$ com herbicida; $\mathrm{S} / \mathrm{H}=$ sem herbicida $)$

\begin{tabular}{|c|c|c|c|c|c|c|}
\hline & \multicolumn{6}{|c|}{ Nutriente } \\
\hline & \multicolumn{2}{|c|}{$\mathrm{N}$ Total $\left(\mathrm{g} \mathrm{kg}^{-1}\right)$} & \multicolumn{2}{|c|}{$\mathrm{P}\left(\mathrm{g} \mathrm{kg}^{-1}\right)$} & \multicolumn{2}{|c|}{$\mathrm{K}\left(\mathrm{g} \mathrm{kg}^{-1}\right)$} \\
\hline & ----- C/H ----- & ----- S/H ------ & ---- C/H ---- & ----- S/H ----- & ----- C/H ----- & ----- S/H ----- \\
\hline Resistente & $7,25 \mathrm{Aa}$ & $7,60 \mathrm{Aa}$ & $0,59 \mathrm{Aa}$ & $0,52 \mathrm{Aa}$ & $37,69 \mathrm{Aa}$ & $37,93 \mathrm{Aa}$ \\
\hline Sensível & $8,45 \mathrm{Aa}$ & $8,39 \mathrm{Aa}$ & $0,51 \mathrm{Aa}$ & $0,62 \mathrm{Aa}$ & $23,39 \mathrm{Aa}$ & $34,54 \mathrm{Aa}$ \\
\hline \multirow[t]{3}{*}{$\mathrm{CV}(\%)$} & \multicolumn{2}{|c|}{33,07} & \multicolumn{2}{|c|}{34,25} & \multicolumn{2}{|c|}{34,78} \\
\hline & \multicolumn{2}{|c|}{$\mathrm{S}\left(\mathrm{g} \mathrm{kg}^{-1}\right)$} & \multicolumn{2}{|c|}{$\mathrm{Ca}\left(\mathrm{g} \mathrm{kg}^{-1}\right)$} & \multicolumn{2}{|c|}{$\mathrm{Mg}\left(\mathrm{g} \mathrm{kg}^{-1}\right)$} \\
\hline & ----- C/H ----- & ----- S/H ----- & ---- C/H ----- & ---- S/H ---- & ---- C/H ---- & ---- S/H ---- \\
\hline Resistente & $1,26 \mathrm{Aa}$ & $0,72 \mathrm{Aa}$ & $1,16 \mathrm{Aa}$ & $0,97 \mathrm{Aa}$ & $1,62 \mathrm{Aa}$ & $1,09 \mathrm{Aa}$ \\
\hline Sensível & $1,12 \mathrm{Aa}$ & $1,21 \mathrm{Aa}$ & $1,09 \mathrm{Aa}$ & $1,37 \mathrm{Aa}$ & $1,17 \mathrm{Aa}$ & $1,31 \mathrm{Aa}$ \\
\hline $\mathrm{CV}(\%)$ & \multicolumn{2}{|c|}{33,22} & \multicolumn{2}{|c|}{24,71} & \multicolumn{2}{|c|}{47,56} \\
\hline
\end{tabular}

Médias seguidas por mesma letra, maiúscula na linha e minúscula na coluna, não diferem entre si pelo teste $\mathrm{F}$ a $5 \%$ de probabilidade 
quando comparado aos demais tratamentos (Tabela 5); também para a concentração total de macronutrientes na planta foram observados maiores valores no biótipo sensivel sem herbicida, em relação ao resistente. Em condições normais, o biótipo sensivel parece apresentar maior taxa de crescimento que o resistente; dessa forma, o biótipo sensível pode ser mais competitivo que o resistente. Esses resultados assemelham-se aos encontrados por Ferreira et al. (2006), que observaram que 35 dias após a emergência o biótipo sensivel apresentava maior número de folhas, altura e massa seca que o resistente.

O biótipo sensivel tratado com herbicida apresentou menores concentrações de $\mathrm{P}, \mathrm{K}$, Ca e Mg $\left(20,91 ; 207,02 ; 10,015\right.$; e 15,24 $\mathrm{g} \mathrm{kg}^{-1}$, respectivamente), comparado ao tratamento em que o produto não foi aplicado $(38,01 ; 411,73$; 15,30; e 21,27 $\mathrm{g} \mathrm{kg}^{-1}$, respectivamente), conforme Tabela 6. Para as concentrações de todos os nutrientes avaliados nas plantas não tratadas ( $\mathrm{N}$ total, $\mathrm{P}, \mathrm{K}, \mathrm{S}, \mathrm{Ca}$ e $\mathrm{Mg}$ ), o biótipo sensivel apresentou maiores valores em comparação ao resistente (Tabela 6). Entretanto, deve-se considerar que o biótipo sensivel em condições normais apresenta maior produção de massa seca que o resistente; desta forma, é esperado que o biótipo sensivel apresente maior concentração de nutrientes.

De acordo com os resultados, pode-se concluir que o biótipo sensivel tem maior capacidade de translocação do glyphosate que o resistente, e os dois biótipos avaliados apresentam exsudação radicular do produto. Com relação ao acúmulo de nutrientes, não houve diferenças entre os biótipos e entre os tratamentos com e sem herbicida nos teores de macronutrientes das raízes. Na presença de herbicida, o biótipo resistente apresentou maior teor de fósforo tanto na parte aérea quanto na planta. Verificou-se tendência de os biótipos resistente e suscetivel tratados com herbicida apresentarem maiores teores de $\mathrm{N}$ total e $\mathrm{N}$ inorgânico na parte aérea e na planta como um todo,

Tabela 4 - Teor de macronutrientes na planta em biótipos de azevém (L. multiflorum) resistentes e sensíveis ao glyphosate, aos 10 DAT $(\mathrm{C} / \mathrm{H}=$ com herbicida; $\mathrm{S} / \mathrm{H}=$ sem herbicida $)$

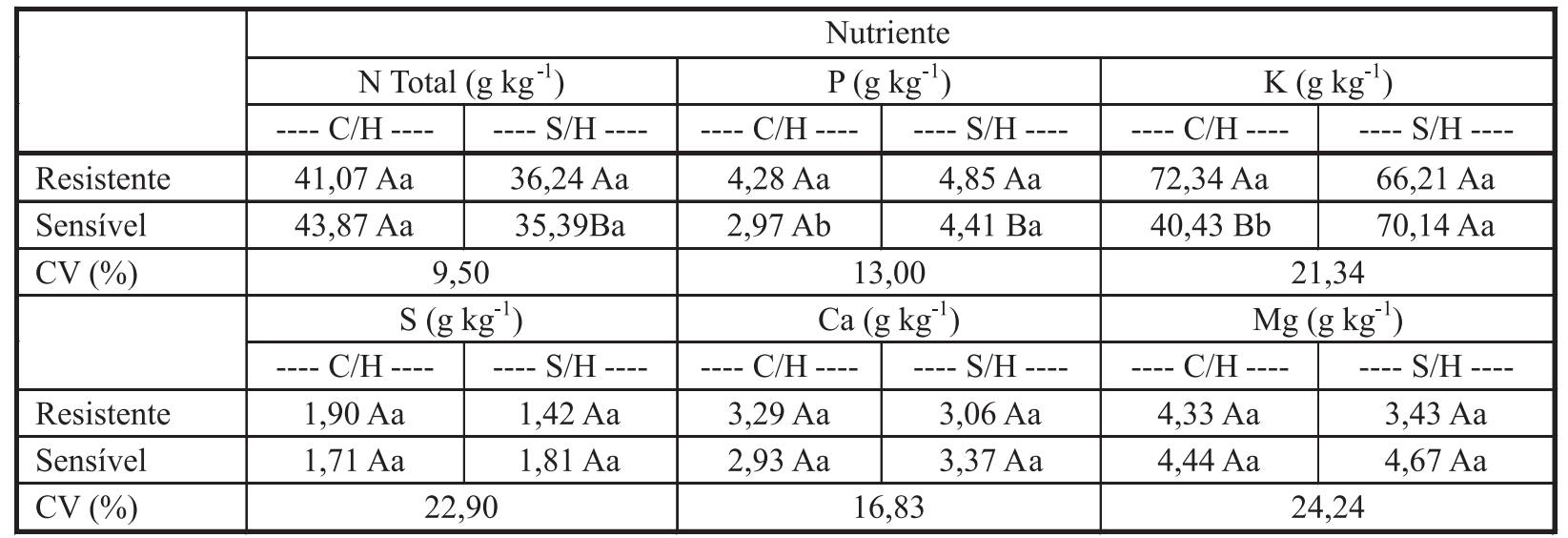

Médias seguidas por mesma letra, maiúscula na linha e minúscula na coluna, não diferem entre si pelo teste $\mathrm{F}$ a $5 \%$ de probabilidade.

Tabela 5 - Massa seca dos biótipos de azevém resistente e sensível com os tratamentos com e sem glyphosate $(\mathrm{C} / \mathrm{H}=$ com herbicida; $\mathrm{S} / \mathrm{H}=$ sem herbicida)

\begin{tabular}{|l|c|c|}
\hline \multirow{2}{*}{} & \multicolumn{2}{|c|}{ Massa seca (g) } \\
\cline { 2 - 3 } & $\mathrm{C} / \mathrm{H}$ & $\mathrm{S} / \mathrm{H}$ \\
\hline Resistente & $3,35 \mathrm{Aa}$ & $3,38 \mathrm{Ab}$ \\
\hline Sensível & $3,51 \mathrm{Ba}$ & $4,53 \mathrm{Aa}$ \\
\hline CV $(\%)$ & \multicolumn{2}{|c|}{10,76} \\
\hline
\end{tabular}

Médias seguidas por mesma letra, maiúscula na linha e minúscula na coluna, não diferem entre si pelo teste $\mathrm{F}$ a $5 \%$ de probabilidade. 
Tabela 6 - Total de macronutrientes na planta em biótipos de azevém (L. multiflorum) resistentes e sensíveis ao glyphosate, aos 15 $\operatorname{DAT}(\mathrm{C} / \mathrm{H}=$ com herbicida; $\mathrm{S} / \mathrm{H}=$ sem herbicida $)$

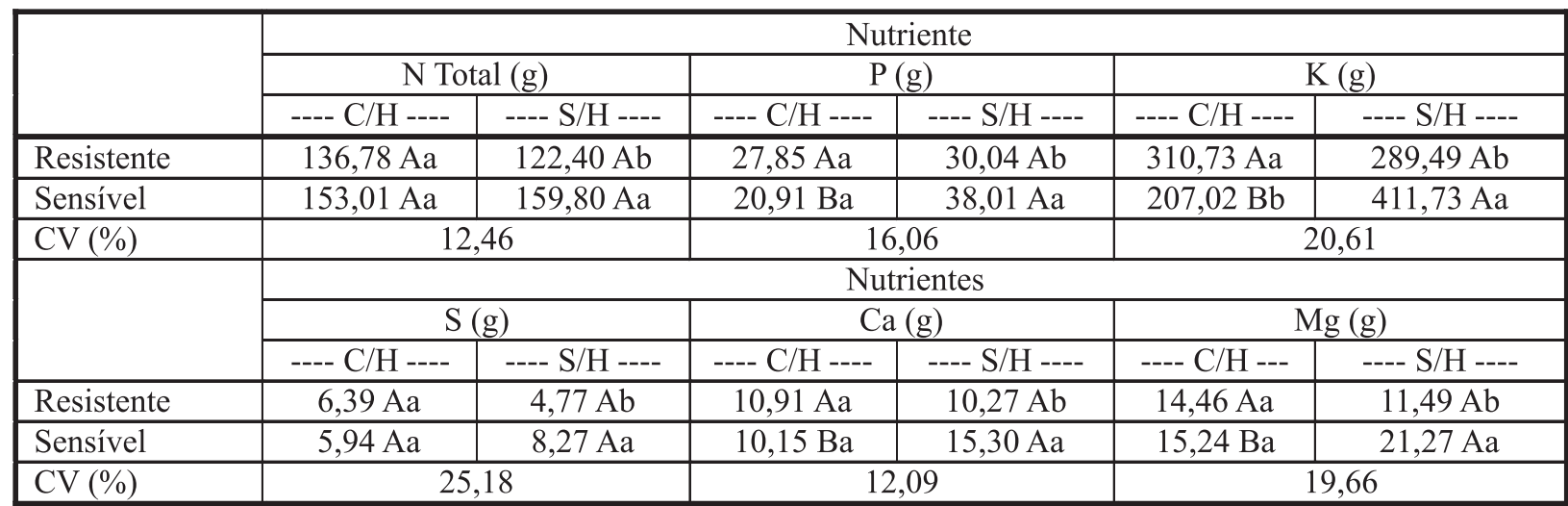

Médias seguidas por mesma letra, maiúscula na linha e minúscula na coluna, não diferem entre si pelo teste $\mathrm{F}$ a $5 \%$ de probabilidade.

quando comparados às plantas não tratadas. Os biótipos não diferiram na capacidade de absorção e acúmulos de macronutrientes na ausência do produto.

\section{LITERATURA CITADA}

BURNSIDE, O. C. Rationale for developing herbicideresistant crops. Weed Tecnhol., v. 6, n. 3, p. 621-25, 1992.

CAROLUS, R. L.; PUTNAM, A. R.; RUIZ, M. R. Effects of soil applied herbicides and caucium on mineral composition of peas (Pisum nativum L.). Hortic. Sci., n. 26, p. $278-283,1971$

CHRISTOFFOLETI, P. J.; LOPEZ-OVEJERO, R. Principais aspectos da resistência de plantas daninhas ao herbicida glyphosate. Planta Daninha, v. 21, n. 3, p. 507$515,2003$.

EASTIN, E. F.; DAVIES, D. E. Effects os atrazine and hidroxitriazine on nitrogen metabolism of selected species. Weed Sci., v. 15, p. 306-309, 1967.

FERREIRA, E. A. et al. Glyphosate no controle de biótipos de azevém e impacto na microbiota do solo. Planta

Daninha, v. 24, n. 3, p. 573-578, 2006.

FERREIRA, E. A. et al. Translocação do glyphosate em biótipos de azevém. Planta Daninha, v. 24, n. 2, p. 365370, 2006.

HESS, F. D. Mechanism of action of inhibitors of amino acid biosynthesis. In: Herbicide action: an intensive course on the activity, selectivity, behavior and fate of herbicides in plants and soil. West Lafayette: Purdue University, 1994. p. 344-365.
HOAGLAND, R. E. Effects os glyphosate on metabolism phenolic compounds. Weed Sci., v. 28, p. 393-400, 1980.

KISSMANN, K. G. Resistência de plantas a herbicidas. São Paulo: Basf Brasileira, 1996. 33 p.

MISHRA, J. S.; KURCHANIA, S. P. Nutrient content in mustard and associated weeds as influenced by nitrogen levels, planting geometry and weed control methods. Indian J. Plant Physiol., v. 6, n. 4, 2001.

PEREZ, A.; KOGAN, M. Glyphosate-resistant Lolium multiflorum in Chilean orchards. Weed Res., v. 43, n. 1, p. $12-19,2002$

RANA, S. S.; ANGIRAS, N. N.; SHARMA, G. D. Effect of herbicides and interculture on nutrient uptake by puddle seeded rice and associated weeds. Indian J. Weed Sci., v. 32, n. 1, p. $70-73,2000$

RODRIGUES, J. J. V.; WORSHAM, A. D.; CORBIN, F. T. Exudation of glyphosate from wheat (Triticum aestivum) plants and its effects on interplanted corn (Zea mays) and soybeans (Glycine max). Weed Sci., v. 30, p. 316-320, 1982

SANTOS, J. B. et al. Avaliação de formulações de glyphosate sobre soja Roundup ready. Planta Daninha, v. 25, n. 1, p. $165-171,2007$.

SILVA, A. A.; VARGAS, L.; FERREIRA, E. A. Herbicidas: resistência de plantas. In: SILVA, A. A.; SILVA, J. F. Tópicos em manejo de plantas daninhas. Viçosa, $\mathrm{MG}$ :

Universidade Federal de Viçosa, 2007. 367 p. 
SWORD, M. A.; TIARKS, A. E.; HAYWOOD, J. D.

Establishment treatments affect relationships among nutrition, productivity and competing vegetation of loblolly pine saplings on a Gulf Coastal Plain site. For. Ecol. Manag., v. 105, n. 2, p.175-188, 1998.

TAIZ, L.; ZEIGER, E. Fisiologia vegetal. 3. ed. São Paulo: Artmed Editora, 2002. 719 p.
VARGAS, L. et al. Alteração das características biológicas dos biótipos de azevém (Lolium multiflorum) ocasionada pela resistência ao herbicida glyphosate. Planta Daninha, v. 23 , n. 1, p.153-160, 2005

WEED SCIENCE. Disponível em: $<$ http://

www.weedscience.org/in.asp $>$. Acesso em: 17 de janeiro de 2007. 